\title{
Density of multivariate homogeneous polynomials on star like domains*
}

\author{
András Kroó ${ }^{\dagger}$
}

July 23, 2019

\begin{abstract}
The famous Weierstrass theorem asserts that every continuous function on a compact set in $\mathbb{R}^{d}$ can be uniformly approximated by algebraic polynomials. A related interesting problem consists in studying the same question for the important subclass of homogeneous polynomials containing only monomials of the same degree. The corresponding conjecture claims that every continuous function on the boundary of convex 0-symmetric bodies can be uniformly approximated by pairs of homogeneous polynomials. The main objective of the present paper is to review the recent progress on this conjecture and provide a new unified treatment of the same problem on non convex star like domains. It will be shown that the boundary of every 0 -symmetric non convex star like domain contains an exceptional zero set so that a continuous function can be uniformly approximated on the boundary of the domain by a sum of two homogeneous polynomials if and only if the function vanishes on this zero set. Thus the Weierstrass type approximation problem for homogeneous polynomials on non convex star like domains amounts to the study of these exceptional zero sets. We will also present an extension of a theorem of Varjú which describes the exceptional zero sets for intersections of star like domains. These results combined with certain transformations of the underlying region will lead to the discovery of some new classes of convex and non convex domains for which the Weierstrass type approximation result holds for homogeneous polynomials.
\end{abstract}

\section{Introduction}

The basic question of approximation theory concerns the possibility of approximation. Is the given family of functions from which we plan to approximate dense in the set of functions we wish to approximate? The first significant density results were those of Weierstrass who proved in 1885 the density of algebraic polynomials in the class of continuous real-valued functions on a compact interval, and the density of trigonometric polynomials in the class of $2 \pi$-periodic continuous real-valued functions. These classical Weierstrass approximation theorems led to numerous generalizations which were applied to other families of functions. They gave rise to the development of a general methods for determining density namely, the Stone-Weierstrass theorem generalizing the above Weierstrass theorem to subalgebras of $C(X), X$ a compact space. In particular, the Stone-Weierstrass theorem yields the multivariate version of the classical Weierstrass theorem asserting that for any compact set $K \subset \mathbb{R}^{d}$ and any continuous real valued function $f \in C(K)$ there is a sequence of polynomials $p_{n} \in P_{n}^{d}$ of degree at most $n$ such that $\lim _{n \rightarrow \infty} p_{n}=f$ uniformly on $K$. Here and in what follows $P_{n}^{d}$ denotes the set of algebraic polynomials of degree at most $n$ in $d$ real variables. For a comprehensive treatment of density results in Approximation theory see the nice survey by Pinkus [?]. Of course, the most interesting density problems correspond to those situations when the subalgebra property fails and thus the Stone-Weierstrass theorem is not applicable. For instance, consider the linear space $M:=\operatorname{span}\left\{x^{\lambda_{j}}, 0=\lambda_{0}<\lambda_{1}<\ldots \uparrow \infty\right\}$. Then $M$ is a linear subspace of $C[0,1]$ which is not a subalgebra, because it is not close relative to multiplication, so the Stone-Weierstrass theorem can not be used. By the famous Müntz theorem $M$ is dense in $C[0,1]$ if and only if $\sum_{j} \frac{1}{\lambda_{j}}=\infty$. Another relevant example is the Lorentz type set of incomplete polynomials $p_{n}(x)=\sum_{n \theta<k<n} a_{k} x^{k}, n \in \mathbb{N}$ where $0<\theta<1$ is a fixed number. This time the set of all these incomplete polynomials is closed relative to multiplication, but clearly it is not linear, i.e., the subalgebra condition fails again. It was shown by G.G. Lorentz, von Golitschek, and Saff and Varga that given $f \in C[0,1]$ there exists a sequence of incomplete polynomials which converges to $f$ uniformly on $[0,1]$ if and only if the function

\footnotetext{
*AMS Subject classification: 41A10, 41A63. Key words and phrases: multivariate homogeneous polynomials, uniform approximation, star like and convex domains

†Supported by the NKFIH - OTKA Grant K111742
} 
vanishes on $\left[0, \theta^{2}\right]$, see [?], pp.86-88. Hence this time in order to compensate for the lack of the subalgebra property one needs to impose an additional restriction that the functions vanish on a certain set. As shown in [?] these exceptional zero sets is typical in general in case when approximating by algebraic polynomials with varying weights. This phenomena of exceptional zero sets will also play a central part in our study of approximation by homogeneous polynomials on 0-symmetric star like domains.

\section{On density of homogeneous polynomials on convex domains}

In this paper we will consider the interesting and difficult problem related to the density of multivariate homogeneous polynomials

$$
H_{n}^{d}:=\left\{\sum_{|\mathbf{k}|=n} a_{\mathbf{k}} \mathbf{x}^{\mathbf{k}}: a_{\mathbf{k}} \in \mathbb{R}\right\}, \mathbf{x} \in \mathbb{R}^{d}, \quad H^{d}:=\cup_{n} H_{n}^{d}
$$

Homogeneous polynomials $h \in H_{n}^{d}$ of degree $n$ contain only monomials of exact degree $n$, and therefore they evidently satisfy the property $h(t \mathbf{x})=t^{n} h(\mathbf{x})$ for every $\mathbf{x} \in \mathbb{R}^{d}$ and $t \in \mathbb{R}$. Hence if $h_{n}(\mathbf{x}), n \in \mathbb{N}$ converge to a nonzero value at some $\mathbf{x} \in \mathbb{R}^{d}$, then they tend to zero at $t \mathbf{x}$ if $|t|<1$ and tend to infinity for $|t|>1$. Thus we must assume that each line that goes through the origin intersects the underlying domain in at most two points. Since homogeneous polynomials are either even or odd depending on their degree it is natural to consider compact sets symmetric with respect to the origin. So in view of the above comments we will restrict our attention to 0-symmetric star like domains $K \subset \mathbb{R}$ which satisfy the property that for every $\mathbf{x} \in K$ we have $(-\mathbf{x}, \mathbf{x}) \in \operatorname{IntK}$ and will study the approximation problem on the boundary $\partial K$ of this 0 -symmetric star like domain $K$. In addition, we clearly need in general both even and odd polynomials in order to approximate arbitrary continuous functions, so the density problem will be considered for sums of pairs of homogeneous polynomials.

Example Consider the unit sphere in $\mathbb{R}^{d}$ given by:

$$
S^{d-1}=\left\{\mathbf{x}=\left(x_{1}, \ldots, x_{d}\right) \in \mathbb{R}^{d}: x_{1}^{2}+\ldots+x_{d}^{2}=1\right\}
$$

It is well known that on $S^{d-1}$ the relation $P_{n}^{d}=H_{n}^{d}+H_{n-1}^{d}$ holds. Now by the Weierstrass theorem $\cup_{n} P_{n}^{d}$ is dense in $C\left(S^{d-1}\right)$. This implies that $H^{d}+H^{d}$ is dense in $C\left(S^{d-1}\right)$, too.

Now we formulate the central conjecture on the density of homogeneous polynomials on convex bodies in $\mathbb{R}^{d}$. (Convex bodies are compact convex sets in $\mathbb{R}^{d}$ with non empty interior.) This conjecture can be regarded as the Weierstrass approximation theorem for homogeneous polynomials.

Conjecture For any 0-symmetric convex body $K \subset \mathbb{R}^{d}$ and every $f \in C(\partial K)$ there exist homogeneous polynomials $h_{2 n}, h_{2 n+1} \in H_{2 n}^{d}, H_{2 n+1}^{d}$ such that uniformly on $\partial K$

$$
f=\lim _{n \rightarrow \infty}\left(h_{2 n}+h_{2 n+1}\right) .
$$

It should be noted that even though the set $H^{d}$ of homogeneous polynomials is closed relative to multiplication and composition nevertheless it is not linear. Nonlinearity of this set means that the Stone-Weierstrass theorem is not applicable off hand in this situation.

The above conjecture has been verified in the following three significant cases:

(i) when $d=2$ for every 0 -symmetric convex body $K \subset \mathbb{R}^{2}$. (This has been done independently by Benko-Kroó [?] and Varjú [?].)

(ii) for any 0 -symmetric convex polytope in $\mathbb{R}^{d}, d \geq 2$. (Varjú [?])

(iii) for any 0 -symmetric regular convex body in $\mathbb{R}^{d}, d \geq 2$ possessing a unique supporting hyperplane at every point on its boundary. (Kroó-Szabados [?])

It is worth noting that statement (ii) corresponding to polytopes is a special case of the following elegant general statement.

Theorem 1 (Varjú [?]) Given any two 0-symmetric star-like domains $K_{1}, K_{2}$ for which the density conjecture (??) holds it follows that the same is true for their intersection $K_{1} \cap K_{2}$, as well.

Another significant contribution to the above conjecture was made by Totik [?] using the concept of $\epsilon$-regularity: a convex body $K \subset \mathbb{R}^{d}$ is called $\epsilon$-regular if the angle between any two normals at every point on its boundary is at most $\epsilon$. Then essentially the following statement can be found in [?]. 
Theorem 2 (Totik [?]) Let $K \subset \mathbb{R}^{d}$ be a 0-symmetric convex body and assume that there exists an integer $\eta_{K}$ depending only on $K$ such that for every $\epsilon>0$ the set $K$ is the intersection of at most $\eta_{K} \epsilon$-regular 0 -symmetric convex bodies. Then the density conjecture (??) holds for $K$.

Theorem 2 allows to recover all cases (i)-(iii) where the density conjecture was shown to be true. Indeed in case (iii) when $K \subset \mathbb{R}^{d}$ is a regular convex body then the assumption of the theorem holds with $\eta_{K}=1(\epsilon=0)$. Furthermore, any 0-symmetric convex polytope in $\mathbb{R}^{d}$ with $2 m$ faces of dimension $d-1$ is an intersection of $m$ 0-symmetric regular convex bodies, so $\eta_{K}=m$ in case (ii). Finally, by Proposition 2 in [?] for any 0-symmetric convex body $K \subset \mathbb{R}^{2}$ we have $\eta_{K}=4$. Clearly this yields the full conjecture in case $d=2$. On the other hand the sufficient condition of Theorem 2 does not hold for some standard convex bodies. For instance, for 0-symmetric circular cones given by the equation

$$
C:=\left\{\mathbf{x}=\left(x_{1}, \ldots, x_{d}\right) \in \mathbb{R}^{d}:\left|x_{1}\right|+\left(x_{2}^{2}+\ldots+x_{d}^{2}\right)^{1 / 2}\right\} \leq 1
$$

the quantity $\eta_{K}$ defined in Theorem 2 is infinite. Nevertheless, it will be shown in Section 5 below that for a wide class of domains of revolution (including circular cones) the density conjecture holds, as well. Thus while Theorem 2 yields a unified approach to treating the known cases (i)-(iii) of the density conjecture, on the other hand it does not cover some standard convex bodies.

In a recent paper Kroó-Totik [?] the density of bivariate homogeneous polynomials was studied on the non convex $L_{\alpha}$ sphere given by

$$
K_{\alpha}:=\left\{(x, y) \in \mathbb{R}^{2}:|x|^{\alpha}+|y|^{\alpha} \leq 1\right\}, \quad 0<\alpha<1
$$

It was verified that $f(x, y)$ is a uniform limit on $\partial K_{\alpha}$ of sums of homogeneous polynomials $h_{2 n}+h_{2 n+1} \in H_{2 n}^{d}+H_{2 n+1}^{d}$ if and only if $f( \pm 1,0)=f(0, \pm 1)=0$, i.e., the function must vanish at the vertices of the $L_{\alpha}$ sphere. This phenomena of an exceptional zero set where the function must vanish in order for homogeneous approximation to hold will be the central theme of this paper.

The main objective of the present paper is to provide a unified treatment of the problem of density of homogeneous polynomials on non convex star like domains. In the next Section 3 it will be shown that the boundary of every 0-symmetric star like domain contains a 0 -symmetric exceptional zero set so that a continuous function can be uniformly approximated on this boundary by a sum of two homogeneous polynomials if and only if the function vanishes on this set. Thus the Weierstrass type approximation problem on non convex star like domains amounts to the study of these exceptional zero sets. In Section 4 we will extend Theorem 1 of Varjú to the case of star like domains with non empty exceptional sets. In Section 5 we will study the exceptional zero sets for bodies of revolution and non convex polytopes. These considerations will allow us to treat in the last Section 6 the density problem on a variety of new domains, including in particular circular cones, as well.

\section{On existence of exceptional zero sets on star like domains}

The density result of [?] for non convex $L_{\alpha}$ sphere with $0<\alpha<1$ quoted above indicates that in order that homogeneous approximation holds for a given continuous function it is necessary and sufficient that the function vanishes at the vertices of the $L_{\alpha}$ sphere. It turns out that such exceptional zero sets exists in general for every 0-symmetric star like domain. We will apply a well known Stone-Weierstrass-type theorem, see e.g. [?, p.13]. For any closed subalgebra of functions $A \subset C(D)$, where $D$ is a compact Hausdorff space denote by $Z_{A}:=\{x \in D:$ $g(x)=0$, for all $g \in A\}$ the zero set of $A$. Then if $A$ separates points in $D \backslash Z_{A}$, it follows that any $f \in C(D)$ which vanishes on $Z_{A}$ belongs to $A$, i.e., $A=\left\{f \in C(D): f=0\right.$ on $\left.Z_{A}\right\}$.

Theorem 3 For every 0-symmetric star like domain $K$ in $\mathbb{R}^{d}$ there exists a 0 -symmetric set $Z(K) \subset \partial K$ so that for any given $f \in C(\partial K)$ the following statements are equivalent

(i) there exist $h_{2 n}+h_{2 n+1} \in H_{2 n}^{d}+H_{2 n+1}^{d}$ such that $f=\lim _{n \rightarrow \infty}\left(h_{2 n}+h_{2 n+1}\right)$ uniformly on $\partial K$

(ii) $f=0$ on $Z(K)$.

Proof. Let $K$ be a 0 -symmetric star like domain in $\mathbb{R}^{d}$. Now denote by $K_{0}$ the set of pairs $\mathbf{z}=(\mathbf{x},-\mathbf{x}), \mathbf{x} \in K$ were these pairs are ordered in such a way that the first nonzero coordinate of $\mathbf{x}$ is positive. The distance in $K_{0}$ is given by $|\mathbf{z}-\mathbf{w}|:=|\mathbf{x}-\mathbf{y}|, \mathbf{z}=(\mathbf{x},-\mathbf{x}), \mathbf{w}=(\mathbf{y},-\mathbf{y}) \in K_{0}$. Clearly $K_{0}$ is compact in this topology. Furthermore, for an even function $f \in C(\partial K)$ set $f_{0}(\mathbf{z})=f(\mathbf{x}), \mathbf{z}=(\mathbf{x},-\mathbf{x}) \in K_{0}$. Then $f_{0} \in C\left(\partial K_{0}\right)$. 
Set

$$
A:=\left\{f \in C(\partial K): f=\lim _{n \rightarrow \infty} h_{2 n}, \quad h_{2 n} \in H_{2 n}^{d}\right\}=\left\{f_{0} \in C\left(\partial K_{0}\right): f_{0}=\lim _{n \rightarrow \infty} h_{2 n}, h_{2 n} \in H_{2 n}^{d}\right\} .
$$

Thus $A$ consists of all limits of even homogeneous polynomials (uniform convergence on $\partial K_{0}$ ). Evidently, all functions in $A$ are even. Let us verify now that $A$ is a closed algebra. Assume $f_{1}, f_{2}, \ldots \in A$ converge to $f$ with an appropriate rate, i.e., $\left|f_{k}-f\right|<1 / k$ on $\partial K$. We need to show that $f \in A$. Let us keep the elements of the polynomial sequence tending to $f_{1}$ up to such an index $N_{2}$ after which elements tending to $f_{2}$ differ by less than $1 / 2$ from $f_{2}$. Then we keep elements tending to $f_{2}$ up to such an $N_{3}>N_{2}$ after which elements tending to $f_{3}$ differ from it by less than $1 / 3$, and so on. Thus after index $N_{k}$ every element of this sequence (defined for every index) will be on distance at most $1 / j \leq 1 / k$ from some $f_{j}(j \geq k)$, which in turn differs by at most $1 / j \leq 1 / k$ from $f$. Hence $A$ is a closed. Linearity of $A$ is obvious. Assume now $f, g \in A$, i.e., $f=\lim _{n \rightarrow \infty} f_{2 n}, g=\lim _{n \rightarrow \infty} g_{2 n}$ with $f_{2 n}, g_{2 n} \in H_{2 n}^{d}$. Then clearly $f_{2 n} \cdot g_{2 n} \in H_{4 n}^{d}, f_{2(n-1)} \cdot g_{2 n} \in H_{4 n-2}^{d}$ and

$$
f_{2 n} \cdot g_{2 n} \rightarrow f g, \quad f_{2(n-1)} \cdot g_{2 n} \rightarrow f g .
$$

Thus $f g \in A$ and we obtain that $A$ is a closed subalgebra in $C\left(\partial K_{0}\right)$.

Denote now $Z_{A}:=\left\{\mathbf{z} \in \partial K_{0}: g(\mathbf{z})=0, \forall g \in A\right\}$. We need to show now that $A$ separates points in $\partial K_{0} \backslash Z_{A}$. Consider distinct $\mathbf{z}=(\mathbf{x},-\mathbf{x}), \mathbf{w}=(\mathbf{y},-\mathbf{y}) \in \partial K_{0} \backslash Z_{A}$. Then $\exists g \in A$ with $g(\mathbf{z})=g(\mathbf{x}) \neq 0$. We have $g=$ $\lim _{n \rightarrow \infty} g_{2 n}$ with some $g_{2 n} \in H_{2 n}^{d}$. Since $\mathbf{x} \neq \mathbf{y}$ and $\mathbf{x} \neq-\mathbf{y}$ there exists a hyperplane $L$ with $\mathbf{y} \in L, \mathbf{x} \in \mathbb{R}^{d} \backslash L$. Hence $\exists h_{2} \in H_{2}^{d}, h_{2}(\mathbf{y})=0, h_{2}(\mathbf{x}) \neq 0$. Clearly $g h_{2}=\lim _{n \rightarrow \infty} g_{2(n-1)} h_{2}, \quad g_{2(n-1)} h_{2} \in H_{2 n}^{d}$. Hence $g h_{2} \in A$ and $g h_{2}(\mathbf{x}) \neq 0, g h_{2}(\mathbf{y})=0$ which means that $g h_{2} \in A$ separates points $\mathbf{z}, \mathbf{w} \in \partial K_{0} \backslash Z_{A}$.

Since $A$ is a closed subalgebra of $C\left(\partial K_{0}\right)$ separating points in $\partial K_{0} \backslash Z_{A}$ by the Stone-Weierstrass type theorem cited above for any $f \in C\left(\partial K_{0}\right)$ with $f=0$ on $Z_{A}$ we have that $f=\lim _{n \rightarrow \infty} h_{2 n}, h_{2 n} \in H_{2 n}^{d}$. This means that any even function $f \in C(\partial K)$ with $f=0$ on $Z_{A}$ is a uniform limit of even homogeneous polynomials on $\partial K$. Note that $Z_{A} \subset \partial K$ is 0 -symmetric.

Now we will verify a similar statement for odd functions. Let $f \in C(\partial K), f=0$ on $Z_{A}$ be odd. Set $g_{i}(\mathbf{x}):=$ $x_{i}|\mathbf{x}|^{-2} f(\mathbf{x}), 1 \leq i \leq d$. Clearly $g_{i} \in C(\partial K), g_{i}=0$ on $Z_{A}$ are even functions. Therefore by the above result for even functions there exist $h_{2 n, i} \in H_{2 n}^{d}, 1 \leq i \leq d$ such that $g_{i}=\lim _{n \rightarrow \infty} h_{2 n, i}, 1 \leq i \leq d$. Then setting

$$
h_{2 n+1}:=\sum_{i} x_{i} h_{2 n, i} \in H_{2 n+1}^{d}
$$

we have uniformly on $\partial K$

$$
h_{2 n+1} \rightarrow \sum_{i} x_{i} g_{i}=f .
$$

Thus the required approximation by homogeneous polynomials holds for both even and odd functions. Now we will use the standard even + odd decomposition to verify the theorem with $Z(K):=Z_{A}$.

$($ ii $) \Rightarrow(i)$. Let $f \in C(\partial K)$ be such that $f=0$ on $Z(K)$. We can write

$$
f=f_{0}+f_{1}, 2 f_{j}(\mathbf{x}):=f(\mathbf{x})+(-1)^{j} f(-\mathbf{x}), j=0,1
$$

where $f_{0}, f_{1} \in C(\partial K)$ are even and odd, respectively. Since $f=0$ on $Z(K)$ and $Z(K)=Z_{A}$ is 0 -symmetric we clearly have that $f_{j}=0$ on $Z_{A}, j=0,1$. Then as shown above

$$
f_{j}=\lim _{n \rightarrow \infty} h_{2 n+j}, \quad h_{2 n+j} \in H_{2 n+j}^{d}, \quad j=0,1
$$

implying $(i)$.

$(i) \Rightarrow($ ii $)$. Since $f=\lim _{n \rightarrow \infty}\left(h_{2 n}+h_{2 n+1}\right)$ we also have that

$$
\left(h_{2 n}+h_{2 n+1}\right)(-\mathbf{x})=h_{2 n}(\mathbf{x})-h_{2 n+1}(\mathbf{x}) \rightarrow f(-\mathbf{x}) .
$$

Therefore $h_{2 n+j} \rightarrow f_{j}, \quad j=0,1$. Hence $f_{0} \in A$ yielding $f_{0}=0$ on $Z_{A}=Z(K)$. We still need to show that $f_{1}=0$ on $Z(K)$. Consider the even function $x_{k} f_{1}(\mathbf{x}), 1 \leq k \leq d$. Then by above $x_{k} h_{2 n+1}(\mathbf{x}) \rightarrow x_{k} f_{1}(\mathbf{x})$ i.e., $x_{k} f_{1}(\mathbf{x}) \in A, 1 \leq k \leq d$. Therefore $x_{k} f_{1}(\mathbf{x})$ must vanish on $Z_{A}=Z(K)$ for every $1 \leq k \leq d$. Since $0 \notin Z(K)$ this obviously implies $f_{1}=0$ on $Z(K)$. 
Clearly, conjecture (??) on density of homogeneous approximation on every convex body can now be reformulated in terms of the exceptional zero sets as follows:

Conjecture. For every 0 -symmetric convex body $K \in \mathbb{R}^{d}$ we have $Z(K)=\emptyset$.

The density results for convex bodies listed in the previous section can be now restated too: $Z(K)=\emptyset$ whenever $K \in \mathbb{R}^{d}$ is a 0 -symmetric convex body which is regular, or is a polytope, or $d=2$. Another related result is given in Varjú [?], Proposition A.1. Essentially, this proposition asserts that there exist 0-symmetric star like domains $K \in \mathbb{R}^{2}$ such that $Z(K)=\partial K$, i.e., homogeneous approximation does not hold anywhere in the domain.

We will explore further the possible structure of exceptional zero sets of 0-symmetric star like domains in Section 4 below. For this end first we will need to extend Varjú's Theorem 1 to the case of 0-symmetric star like domains with nonempty exceptional zero sets. This is accomplished in the next Section 3.

\section{On exceptional zero sets for intersection of star like domains}

According to the result of Varjú [?] cited above whenever $K_{1}, K_{2}$ are 0-symmetric star-like domains in $\mathbb{R}^{d}$ for which density of homogeneous polynomials holds (i.e. $Z\left(K_{1}\right)=Z\left(K_{1}\right)=\emptyset$ ) then the same is true for $K_{1} \cap K_{2}$. In other words in terms of the exceptional zero sets we have the following implication

$$
Z\left(K_{1}\right)=Z\left(K_{2}\right)=\emptyset \Rightarrow Z\left(K_{1} \cap K_{2}\right)=\emptyset .
$$

Now we would like to adapt Varjú's result to a more general setting of star like domains with non empty exceptional zero sets. In order to accomplished this we will use the elegant construction given by Varjú in [?]. But needless to say in the presence of non empty exceptional zero sets this construction becomes technically more complicated.

Theorem 4 Let $K_{1}, K_{2}$ be any 0 -symmetric star-like domains in $\mathbb{R}^{d}$. Then setting $K:=K_{1} \cap K_{2}$ we have

$$
Z(K) \subset\left(Z\left(K_{1}\right) \cup Z\left(K_{2}\right)\right) \cap K
$$

Proof. We may assume without the loss of generality that $K_{1} \cup K_{2} \subset B(0,1 / 4)$. Denote by $I^{2}:=\{(x, y):$ $\max \{|x|,|y|\}=1\}$ the unit square on the 2-dimensional plane and set

$$
\Omega_{\delta}:=\left\{(x, y) \in I^{2}: x, y \geq-\delta\right\}, \delta>0
$$

First we need to note that for any $0<\delta<1$ and $0<\epsilon<\delta$ there exist bivariate homogeneous polynomials $H_{k} \in H_{k}^{2}, k=m, m+1, m \geq 4$ such that

$$
\left|1-H_{k}(x, y)\right| \leq \epsilon, \quad(x, y) \in \Omega_{\delta}, \quad k=m, m+1, \quad \text { and }\left|H_{k}(x, y)\right| \leq 2, \quad(x, y) \in I^{2} .
$$

Indeed, if $m$ is even the above claim follows from the fact that $\Omega_{\delta} \subset I^{2}$ and even homogeneous polynomials can approximate 1 uniformly on $I^{2}$. For $m+1$ odd we can consider an odd continuous function $g$ on $I^{2}$ such that $g=1$ on $\Omega_{\delta}$, such functions evidently exist. Since any odd continuous function on $I^{2}$ is a uniform limit of odd homogeneous polynomials on $I^{2}$ relation (??) follow in this case, as well.

Let us consider the nonnegative continuous function

$$
f(\mathbf{x}):=\operatorname{dist}\left(\mathbf{x}, Z\left(K_{1}\right) \cup Z\left(K_{2}\right)\right) \in C\left(\mathbb{R}^{d}\right) .
$$

Clearly,

$$
f=0 \text { on } Z\left(K_{1}\right) \cup Z\left(K_{1}\right) \text {, and } 0<f \leq \frac{1}{2} \text { on }\left(K_{1} \cup K_{2}\right) \backslash\left(Z\left(K_{1}\right) \cup Z\left(K_{1}\right)\right) .
$$

In addition, since $Z\left(K_{1}\right) \cup Z\left(K_{2}\right)$ is 0 -symmetric it follows that $f$ is an even function. Therefore by Theorem 1 there exist even homogeneous polynomials $R_{2 n, i} \in H_{2 n}^{d}$ such that for $n>n_{0}$

$$
-R_{2 n, i}(\mathbf{x}) \leq\left|R_{2 n, i}(\mathbf{x})-f(\mathbf{x})^{\frac{1}{2 m}}\right| \leq \frac{\epsilon}{m}, \quad \mathbf{x} \in \partial K_{i}, i=1,2
$$

Now consider the homogeneous polynomials of degree $2(n+k) m+2 k$ defined by

$$
Q_{2(n+k) m+2 k}(\mathbf{x}):=H_{m}\left(R_{2 n, 1}(\mathbf{x}), R_{2 n, 2}(\mathbf{x})\right) H_{m+1}\left(R_{2 k, 1}(\mathbf{x}), R_{2 k, 2}(\mathbf{x})\right), \quad n>n_{0}, n_{0}<k \leq n_{0}+m .
$$


Clearly, for any $n_{0}$ all large even integers are of the form $2(n+k) m+2 k$ with $n \geq n_{0}, n_{0}<k \leq n_{0}+m$.

We are going to show now that $Q_{2(n+k) m+2 k} \rightarrow f, n \rightarrow \infty$ uniformly on $\partial K$. Since $f>0$ on $\mathbb{R}^{d} \backslash\left(Z\left(K_{1}\right) \cup Z\left(K_{2}\right)\right)$ in view of Theorem 1 this evidently yields $Z(K) \subset\left(Z\left(K_{1}\right) \cup Z\left(K_{2}\right)\right)$ which is the claim of the theorem. For this end it suffices to show that for any $n_{0}$ large enough

$$
\left\|f^{\frac{1}{2}}-H_{m}\left(R_{2 n, 1}, R_{2 n, 2}\right)\right\|_{\partial K}<\epsilon,\left\|f^{\frac{1}{2}}-H_{m+1}\left(R_{2 k, 1}, R_{2 k, 2}\right)\right\|_{\partial K}<\epsilon, \quad n, k>n_{0} .
$$

We will prove the first estimate above, the second can be verified analogously.

Consider any $\mathbf{x} \in \partial K$. We may assume, for instance that $\mathbf{x} \in \partial K_{1} \cap K_{2}$, i.e., $\alpha \mathbf{x} \in \partial K_{2}$ for some $\alpha \geq 1$. Set

$$
\mu_{n}(\mathbf{x}):=\max \left\{R_{2 n, 1}(\mathbf{x}), R_{2 n, 2}(\mathbf{x})\right\} .
$$

We shall distinguish now between two cases depending on the value of $\mu_{n}(\mathbf{x})$.

Case 1. $\mu_{n}(\mathbf{x}) \leq 0$. Then by (??) we have $-\frac{\epsilon}{m} \leq R_{2 n, i}(\mathbf{x}) \leq 0$, i.e., $\left|R_{2 n, i}(\mathbf{x})\right| \leq \frac{\epsilon}{m}, i=1,2$. Thus using again

$$
f(\mathbf{x}) \leq\left(\left|R_{2 n, 1}(\mathbf{x})\right|+\frac{\epsilon}{m}\right)^{2 m} \leq\left(\frac{2 \epsilon}{m}\right)^{2 m} \leq \frac{\epsilon}{2} .
$$

Furthermore, since $\left|H_{k}\right| \leq 2$ on $I^{2}$ it follows that

$$
\left|H_{m}\left(R_{2 n, 1}(\mathbf{x}), R_{2 n, 2}(\mathbf{x})\right)\right|=\left(\frac{\epsilon}{m}\right)^{m}\left|H_{m}\left(\frac{m}{\epsilon} R_{2 n, 1}(\mathbf{x}), \frac{m}{\epsilon} R_{2 n, 2}(\mathbf{x})\right)\right| \leq 2\left(\frac{\epsilon}{m}\right)^{m} \leq \frac{\epsilon}{2} .
$$

Hence by the last two estimates

$$
\left|f(\mathbf{x})-H_{m}\left(R_{2 n, 1}(\mathbf{x}), R_{2 n, 2}(\mathbf{x})\right)\right| \leq \epsilon .
$$

Case 2. $\mu_{n}(\mathbf{x})>0$. Now we set

$$
z_{n}(\mathbf{x}):=\frac{1}{\mu_{n}(\mathbf{x})}\left(R_{2 n, 1}(\mathbf{x}), R_{2 n, 2}(\mathbf{x})\right) \in \mathbb{R}^{2} .
$$

Recall that by (??)

$$
-\delta<-\frac{\epsilon}{m} \leq R_{2 n, i}(\mathbf{x})
$$

and therefore we clearly have $z_{n}(\mathbf{x}) \in \Omega_{\delta}$. Thus by (??)

$$
-\epsilon+1 \leq H_{k}\left(z_{n}(\mathbf{x})\right) \leq \epsilon+1, \quad k=m, m+1 .
$$

Since $H_{m}$ is a homogeneous polynomial of degree $m$ we have

$$
H_{m}\left(R_{2 n, 1}(\mathbf{x}), R_{2 n, 2}(\mathbf{x})\right)=\mu_{n}(\mathbf{x})^{m} H_{m}\left(z_{n}(\mathbf{x})\right) .
$$

Moreover, using (??) for $\mathbf{x} \in \partial K_{1}$ and $\alpha \mathbf{x} \in \partial K_{2}$ we have for sufficiently large $n$

$$
-\frac{\epsilon}{m}+f^{\frac{1}{2 m}}(\mathbf{x}) \leq R_{2 n, 1}(\mathbf{x}) \leq \frac{\epsilon}{m}+f^{\frac{1}{2 m}}(\mathbf{x}),-\frac{\epsilon}{m}+f^{\frac{1}{2 m}}(\alpha \mathbf{x}) \leq R_{2 n, 2}(\alpha \mathbf{x}) \leq \frac{\epsilon}{m}+f^{\frac{1}{2 m}}(\alpha \mathbf{x}) .
$$

Obviously, since $f$ is continuous and $K_{2}$ is a compact domain we can choose a sufficiently small $\alpha_{\epsilon}>0$ independent of $\mathbf{x}$ so that whenever $1 \leq \alpha<1+\alpha_{\epsilon}$ we have

$$
\left|f^{\frac{1}{2 m}}(\alpha \mathbf{x})-f^{\frac{1}{2 m}}(\mathbf{x})\right| \leq \frac{\epsilon}{m}
$$

This and the second inequality in (??) yield

$$
R_{2 n, 2}(\mathbf{x})=\alpha^{-2 n} R_{2 n, 2}(\alpha \mathbf{x}) \leq \frac{2 \epsilon}{m}+f^{\frac{1}{2 m}}(\mathbf{x})
$$

Thus combining the above estimate with the first inequality in (??) we have whenever $\alpha<1+\alpha_{\epsilon}$

$$
-\frac{\epsilon}{m}+f^{\frac{1}{2 m}}(\mathbf{x}) \leq \mu_{n}(\mathbf{x})=\max \left\{R_{2 n, 1}(\mathbf{x}), R_{2 n, 2}(\mathbf{x})\right\} \leq \frac{2 \epsilon}{m}+f^{\frac{1}{2 m}}(\mathbf{x})
$$


that is

$$
\left|\mu_{n}(\mathbf{x})-f^{\frac{1}{2 m}}(\mathbf{x})\right| \leq \frac{2 \epsilon}{m} .
$$

Since $|f|,\left|R_{2 n, i}\right| \leq 1$ on $K_{i}, i=1,2$ this easily yields when $1 \leq \alpha<1+\alpha_{\epsilon}$

$$
\left|\mu_{n}(\mathbf{x})^{m}-f^{\frac{1}{2}}(\mathbf{x})\right| \leq 2 \epsilon .
$$

Let us show that similar estimate holds for $\alpha>1+\alpha_{\epsilon}$, as well. Indeed, in this case we have

$$
\left|R_{2 n, 2}(\mathbf{x})\right|=\alpha^{-2 n}\left|R_{2 n, 2}(\alpha \mathbf{x})\right| \leq \alpha^{-2 n} \leq\left(1+\alpha_{\epsilon}\right)^{-2 n}<\epsilon, \quad n>n_{0} .
$$

In addition, as above by the first inequality in (??)

$$
-\epsilon+f^{\frac{1}{2}}(\mathbf{x}) \leq R_{2 n, 1}^{m}(\mathbf{x}) \leq \epsilon+f^{\frac{1}{2}}(\mathbf{x}) .
$$

Hence by the last two estimates we get for $n>n_{0}$

$$
-\epsilon+f^{\frac{1}{2}}(\mathbf{x}) \leq R_{2 n, 1}^{m}(\mathbf{x}) \leq \mu_{n}^{m}(\mathbf{x})=\max \left\{R_{2 n, 1}^{m}(\mathbf{x}), R_{2 n, 2}^{m}(\mathbf{x})\right\} \leq \max \left\{\epsilon+f^{\frac{1}{2}}(\mathbf{x}), \epsilon\right\} \leq 2 \epsilon+f^{\frac{1}{2}}(\mathbf{x}),
$$

i.e., estimate (??) holds for every $\alpha \geq 1$.

Finally, applying estimates (??) and (??) for the homogeneous polynomial (??) we easily arrive at

$$
f^{\frac{1}{2}}(\mathbf{x})-5 \epsilon \leq H_{m}\left(R_{2 n, 1}(\mathbf{x}), R_{2 n, 2}(\mathbf{x})\right) \leq f^{\frac{1}{2}}(\mathbf{x})+5 \epsilon,
$$

where the last estimates hold for any $\mathbf{x} \in \partial\left(K_{1} \cap K_{2}\right)$ with $n>n_{0}$ independent of $\mathbf{x} \square$.

Of course, Varjú's result according to which condition $Z\left(K_{1}\right)=Z\left(K_{2}\right)=\emptyset$ implies that $Z\left(K_{1} \cap K_{2}\right)=\emptyset$, i.e., the density of homogeneous polynomials on $\partial\left(K_{1} \cap K_{2}\right)$ is now a special case of Theorem 4 . Moreover, Theorem 4 yields an essentially more general conclusion that the same statement is true if we replace $Z\left(K_{1}\right)=Z\left(K_{2}\right)=\emptyset$ by a substantially weaker assumption $Z\left(K_{1}\right) \cap K_{2}=\emptyset, Z\left(K_{2}\right) \cap K_{1}=\emptyset$.

Corollary 5 Let $K_{1}, K_{2}$ be any 0 -symmetric star-like domains in $\mathbb{R}^{d}$ such that $Z\left(K_{1}\right) \cap K_{2}=\emptyset, Z\left(K_{2}\right) \cap K_{1}=\emptyset$. Then $Z\left(K_{1} \cap K_{2}\right)=\emptyset$.

Various applications of Theorem 4 and Corollary 5 will be presented in Section 5 below.

\section{On exceptional zero sets for bodies of revolution and non convex polytopes}

We will introduce now a general method of deriving new classes of 0-symmetric star like domains satisfying the homogeneous density property (??) which is based on rotation. This rotation will require that the domain is also symmetric with respect to one of the coordinate axises. Obviously, for this purpose we can choose any of the coordinates, but for the simplicity of the exposition throughout this section the last coordinate will be chosen as the axis of symmetry.

Thus we consider a 0 -symmetric star like domain $D \subset \mathbb{R}^{k}, k \geq 2$ which, in addition, is assumed to be symmetric with respect to the last coordinates $x_{k}$, i.e., $\left(x_{1}, \ldots, x_{k}\right) \in D \Leftrightarrow\left(x_{1}, \ldots, x_{k-1},-x_{k}\right) \in D$.

Consider the mapping $T: \mathbb{R}^{d} \rightarrow \mathbb{R}_{+}^{k}, 2 \leq k \leq d-1$ defined by

$$
T(\mathbf{x}):=\left(x_{1}, \ldots, x_{k-1},\left(x_{k}^{2}+\ldots+x_{d}^{2}\right)^{1 / 2}\right), \quad \mathbf{x}=\left(x_{1}, \ldots, x_{d}\right) \in \mathbb{R}^{d} .
$$

Then the rotation of the set $D$ around the corresponding axis of symmetry yields the following domain of revolution

$$
K_{D}:=\left\{\mathbf{x} \in \mathbb{R}^{d}: T(\mathbf{x}) \in D\right\} \subset \mathbb{R}^{d} .
$$

It can be easily verified that under the above assumptions on $D$ the body of revolution $K_{D}$ is a 0 -symmetric star like domain in $\mathbb{R}^{d}$. Indeed, if $\mathbf{x} \in K_{D}$ then by (??) $T(\mathbf{x}) \in D$. Since $D$ is 0 -symmetric this implies

$$
-T(\mathbf{x})=\left(-x_{1}, \ldots,-x_{k-1},-\left(x_{k}^{2}+\ldots+x_{d}^{2}\right)^{1 / 2}\right) \in D .
$$


Moreover, using also that $D$ is symmetric with respect to $x_{k}$ we obtain

$$
T(-\mathbf{x})=\left(-x_{1}, \ldots,-x_{k-1},\left(x_{k}^{2}+\ldots+x_{d}^{2}\right)^{1 / 2}\right) \in D
$$

i.e., $K_{D}$ is a 0 -symmetric star like domain. Clearly we also have

$$
\partial K_{D}=\left\{\mathbf{x} \in \mathbb{R}^{d}: T(\mathbf{x}) \in \partial D\right\} .
$$

By Theorem 3 of Section 2 domain $D$ possesses a corresponding exceptional zero set $Z(D)$ which controls which continuous functions can be approximated by homogeneous polynomials uniformly on $\partial D$. It is natural to expect, that the exceptional zero set of the domain of revolution $K_{D}$ given by (??) is just the rotation of $Z(D)$ around the axis of symmetry. Our next assertion shows that indeed this is the case.

Theorem 6 Let $D$ be a 0-symmetric star-like domain in $\mathbb{R}^{k}, k \geq 2$, which in addition is also symmetric with respect to the last coordinate $x_{k}$. Consider the domain of revolution $K_{D} \in \mathbb{R}^{d}, d \geq k+1$ given by (??). Then

$$
Z\left(K_{D}\right)=\left\{\boldsymbol{x} \in \partial K_{D}: T(\boldsymbol{x}) \in Z(D)\right\} .
$$

Proof. Set $Z^{*}:=\left\{\mathbf{x} \in \partial K_{D}: T(\mathbf{x}) \in Z(D)\right\}$. Let us verify first that $Z^{*} \subset Z\left(K_{D}\right)$. Consider any $\mathbf{a}:=\left(a_{1}, \ldots, a_{d}\right) \in$ $Z^{*}$, i.e., $T(\mathbf{a}) \in Z(D)$. Assume that for some $f \in C\left(\partial K_{D}\right)$ we have that $f=\lim _{n \rightarrow \infty}\left(h_{2 n}+h_{2 n+1}\right)$ uniformly on $\partial K_{D}$ where $h_{2 n}+h_{2 n+1} \in H_{2 n}^{d}+H_{2 n+1}^{d}$. Set $\mathbf{b}:=\left(a_{k}^{2}+\ldots+a_{d}^{2}\right)^{-1 / 2}\left(0, \ldots, 0, a_{k}, \ldots, a_{d}\right) \in \mathbb{R}^{d}$, and consider the $k$-dimensional plane in $\mathbb{R}^{d}$

$$
M_{k}:=\operatorname{span}\left\{\mathbf{e}_{1}, \ldots, \mathbf{e}_{\mathrm{k}-1}, \mathbf{b}\right\} \subset \mathbb{R}^{\mathrm{d}}
$$

with $\mathbf{e}_{j}, 1 \leq j \leq d$ being the standard orthonormal basis in $\mathbb{R}^{d}$. Then evidently,

$$
K_{D} \cap M_{k}=\left\{\left(x_{1}, \ldots, x_{k}\right) \in \mathbb{R}^{k}:\left(x_{1}, \ldots, x_{k-1},\left|x_{k}\right|\right) \in D\right\}=D, \partial K_{D} \cap M_{k}=\partial D
$$

where the symmetry of $D$ with respect to the last coordinate $x_{k}$ was used above. Furthermore, denoting the restriction of homogeneous polynomials $h_{2 n}, h_{2 n+1} \in H_{2 n}^{d}, H_{2 n+1}^{d}$ and $f \in C\left(\partial K_{D}\right)$ to the $k$ dimensional plane $M_{k}$ by $r_{2 n}, r_{2 n+1} \in H_{2 n}^{k}, H_{2 n+1}^{k}$ and $g \in C\left(\partial K_{D} \cap M_{k}\right)$, respectively we have $g=\lim _{n \rightarrow \infty}\left(r_{2 n}+r_{2 n+1}\right)$ uniformly on $\partial K_{D} \cap M_{k}=\partial D$. Since $T(\mathbf{a}) \in Z(D)$ it follows that $f(\mathbf{a})=g(T(\mathbf{a}))=0$. This means that $\mathbf{a} \in Z\left(K_{D}\right)$ and therefore $Z^{*} \subset Z\left(K_{D}\right)$.

Now consider any $\mathbf{a}:=\left(a_{1}, \ldots, a_{d}\right) \in \partial K_{D} \backslash Z^{*}$. Then $T(\mathbf{a}) \in \partial D \backslash Z(D)$. Therefore by Theorem 3 we can choose a function $g \in C(\partial D)$ to be even in variable $x_{k}$ such that $g(T(\mathbf{a}))>0$ and $g=\lim _{n \rightarrow \infty}\left(r_{2 n}+r_{2 n+1}\right)$ uniformly on $\partial D$ for proper $r_{2 n}+r_{2 n+1} \in H_{2 n}^{k}+H_{2 n+1}^{k}$. Since $D$ is symmetric with respect to the last coordinate $x_{k}$ and $g \in C(\partial D)$ is even in variable $x_{k}$ without the loss of generality it can be assumed that $r_{2 n}$ and $r_{2 n+1}$ contain only even powers of $x_{k}$. With this in mind we clearly have that

$$
h_{j}(\mathbf{x}):=r_{j}(T(\mathbf{x}))=r_{j}\left(\left(x_{1}, \ldots, x_{k-1},\left(x_{k}^{2}+\ldots+x_{d}^{2}\right)^{1 / 2}\right)\right) \in H_{j}^{d}, \quad j=2 n, 2 n+1, \quad \mathbf{x} \in \mathbb{R}^{d} .
$$

Now setting $f(\mathbf{x})=g(T(\mathbf{x}))=0$ it obviously follows that $f=\lim _{n \rightarrow \infty}\left(h_{2 n}+h_{2 n+1}\right)$ uniformly on $\partial K_{D}$ where $f(\mathbf{a})=g(T(\mathbf{a}))>0$. Thus $\mathbf{a} \in \partial K_{D} \backslash Z\left(K_{D}\right)$ yielding $Z\left(K_{D}\right) \subset Z^{*}$ and therefore $Z\left(K_{D}\right)=Z^{*}$.

Corollary 7 Let $D$ be any 0-symmetric star-like domains in $\mathbb{R}^{k}, k \geq 2$ which in addition is symmetric with respect to the last coordinate $x_{k}$. Assume that the density conjecture (??) holds for D. Then the homogeneous approximation property (??) is true for the domain of revolution $K_{D}$, as well.

Denote by $B^{d}(\mathbf{x}, r)$ the ball in $\mathbb{R}^{d}$ of radius $r$ centered at $\mathbf{x}$, and let $S^{d-1}:=\partial B^{d}(0,1)$ be the unit sphere. Given a 0 -symmetric star like domain $K$ we will say that it is locally convex at a boundary point $\mathbf{x}_{0} \in \partial K$ if $B^{d}\left(\mathbf{x}_{0}, \epsilon\right) \cap K$ is convex for some sufficiently small $\epsilon>0$. Now consider the set of all non locally convex points of $\partial K$ given by

$$
\partial K^{*}:=\left\{\mathbf{x} \in \partial K: B^{d}(\mathbf{x}, \epsilon) \cap K \text { is not convex for any } \epsilon>0\right\} .
$$

In case when $K \subset \mathbb{R}^{d}$ is a 0 -symmetric star like polytope its boundary is a subset of a finite union of $d-1$ dimensional hyper planes in $\mathbb{R}^{d}$ and $\partial K^{*}$ is the union all "inner" $d$-2-dimensional faces of the polytope $K$. Recall that by Varjú's theorem $Z(K)=\emptyset$ if $K$ is convex. Obviously, $\partial K^{*}=\emptyset$ if and only if $K$ is a convex polytope. Now we are going to show that $\partial K^{*}$ is always contained in the exceptional zero set of the polytope $K$ which in particular implies that the convexity of the polytope is necessary and sufficient for the homogeneous density condition (??) to hold. 
Theorem 8 Let $K$ be a 0-symmetric star like polytope in $\mathbb{R}^{d}, d \geq 2$. Then $\partial K^{*} \subset Z(K)$. Consequently, $Z(K)=\emptyset$ if and only if $K$ is convex.

Proof. Assume that $\partial K^{*} \neq \emptyset$ and consider any $\mathbf{x}_{0} \in \partial K^{*}$. We claim that for some $d-1$ dimensional plane $L$ passing through $\mathbf{x}_{0}$ and the origin we have that $\mathbf{x}_{0} \in \partial K_{L}^{*}$ with $K_{L}:=K \cap L$ i.e., $\mathbf{x}_{0}$ is a non locally convex point of $\partial K_{L}$, as well. Indeed, let $u_{L} \in S^{d-1}$ be the normal of $L$ and denote by $r_{L}$ the radius of the largest ball in $L$ so that $B^{d-1}\left(\mathbf{x}_{0}, r_{L}\right) \cap K_{L}$ is convex. Obviously, $r_{L}: S^{d-1} \rightarrow \mathbb{R}^{+}$is a continuous nonnegative function whose domain is a compact subset of $S^{d-1}$ which therefore attains its minimum $r_{0}$. If $r_{0}>0$ then clearly $B^{d}\left(\mathbf{x}_{0}, r_{0}\right) \cap K$ is convex, in contradiction with $\mathbf{x}_{0} \in \partial K^{*}$. Thus $r_{L}=0$ for some $d-1$ dimensional plane $L$ and proceeding by induction it follows that there exists a 2-dimensional plane $L_{2}$ containing $\mathbf{x}_{0}$ and the origin, so that setting $K_{2}:=K \cap L_{2}$ we have $\mathbf{x}_{0} \in \partial K_{2}^{*}$, i.e., $\mathbf{x}_{0} \in \partial K_{2}$ is an inner vertex of the polygon $K_{2}$. Thus without the loss of generality we may assume that for the polygon $K_{2} \subset \mathbb{R}^{2}$ we have $\left[A, \mathbf{x}_{0}\right] \cup\left[\mathbf{x}_{0}, B\right] \subset \partial K_{2}$ with some

$$
\mathbf{x}_{0}=(0, d), \quad A=(-\delta, a), \quad B=(\delta, b), \quad \delta>0, a>d>0, \quad b>d>0 .
$$

Assume now that contrary to the claim of the theorem $\mathbf{x}_{0} \notin Z(K)$. Consider the function $f(\mathbf{x}):=\operatorname{dist}(\mathbf{x}, Z(K)) \in$ $C\left(\mathbb{R}^{d}\right)$. Note that since $Z(K)$ is 0 -symmetric the function $f$ is even. Obviously, $f=0$ on $Z(K)$ and hence by Theorem 3 there exist $h_{2 n} \in H_{2 n}^{2}$ such that $f=\lim _{n \rightarrow \infty} h_{2 n}$ uniformly on $\partial K_{2}$. Furthermore since $\mathbf{x}_{0} \notin Z(K)$ we have $f\left(\mathbf{x}_{0}\right)>0$ for this $\mathbf{x}_{0} \in \partial K_{2}$. Then $h_{2 n} \in H_{2 n}^{2}$ are uniformly bounded on $\partial K_{2}$, i.e. $\left\|h_{2 n}\right\|_{\partial K_{2}} \leq M, \forall n$ with some $M>0$ and $\left|h_{2 n}\left(\mathbf{x}_{0}\right)\right|>\frac{f\left(\mathbf{x}_{0}\right)}{2}>0$ for $n$ sufficiently large. Set now $\mathbf{x}_{t}:=(t, d)$. Then by the star like property of $K_{2}$ it follows that $\mathbf{x}_{t} \in K_{2}$ whenever $0 \leq|t| \leq t_{0}$ with a sufficiently small $t_{0}$. Furthermore, with some $\alpha_{t}>1$ we have that $\mathbf{y}_{t}=\alpha_{t} \mathbf{x}_{t} \in\left[\mathbf{x}_{0}, B\right]$. Then it is easy to see that $\left|\mathbf{x}_{t}-\mathbf{y}_{t}\right| \geq c t$ with some $c>0$, i.e., $\alpha_{t}>1+c_{1} t, 0 \leq t \leq t_{0}$. Thus we obtain for $h_{2 n} \in H_{2 n}^{2}$ and $\mathbf{y}_{t}=\alpha_{t} \mathbf{x}_{t} \in\left[\mathbf{x}_{0}, B\right] \subset \partial K_{2}$

$$
\left|h_{2 n}\left(\mathbf{x}_{t}\right)\right|=\left|h_{2 n}\left(\alpha_{t}^{-1} \mathbf{y}_{t}\right)\right|=\alpha_{t}^{-2 n}\left|h_{2 n}\left(\mathbf{y}_{t}\right)\right| \leq M\left(1+c_{1} t\right)^{-2 n} \leq M e^{-c_{2} n t}, 0 \leq t \leq t_{0} .
$$

Repeating the same argument for the segment $\left[A, \mathbf{x}_{0}\right] \subset \partial K_{2}$ yields with some $c_{3}>0$

$$
\left|h_{2 n}\left(\mathbf{x}_{t}\right)\right| \leq M e^{c_{3} n t}, \quad-t_{0} \leq t \leq 0 .
$$

Thus combining the last two estimates we obtain with some $C>0$

$$
\left|h_{2 n}\left(\mathbf{x}_{t}\right)\right| \leq M e^{-C n|t|},|t| \leq t_{0},
$$

where evidently $h_{2 n}\left(\mathbf{x}_{t}\right)=h_{2 n}(t, d):=p_{2 n}(t)$ is a univariate algebraic polynomial of degree at most $2 n$ satisfying

$$
p_{2 n}(0)=h_{2 n}(0, d)=h_{2 n}\left(\mathbf{x}_{0}\right)>\frac{f\left(\mathbf{x}_{0}\right)}{2}>0 .
$$

Thus univariate polynomials $q_{2 n}(t):=p_{2 n}(0)^{-1} p_{2 n}(t)$ will satisfy the conditions

$$
q_{2 n}(0)=1, \quad\left|q_{2 n}(t)\right| \leq C_{1} e^{-C n|t|}, ;|t| \leq T, ; n \in \mathbb{N},
$$

with $C_{1}:=\frac{2 M}{f\left(\mathbf{x}_{0}\right)}$. But this rate of decrease of polynomials $q_{2 n}$ contradicts a fundamental result on fast decreasing polynomials proved by Ivanov-Totik [?] because it is shown in [?] that $q_{2 n}(0)=1,\left|q_{2 n}(t)\right| \leq C_{1} e^{-C n \phi(t)},|t| \leq$ $T, n \in \mathbb{N}$ can hold with some $\phi$ if and only if $\int_{0}^{1} t^{-2} \phi(t) d t<\infty$. Thus $\partial K^{*} \subset Z(K)$ which is the first statement of the theorem. In particular this also implies that $Z(K) \neq \emptyset$ if $K$ is not convex. This together with Varjú's result for convex polytopes yields the second claim of the theorem.

\section{New classes of convex and star like domains satisfying the density property}

In this final part of the paper we will provide various applications of the results from previous sections which lead to essentially new types of convex or star like domains for which the the homogeneous approximation property (??) is fulfilled. 
A. "Nowhere convex" star like domains with homogeneous approximation property. As it was mentioned above in [?] the authors considered the density of bivariate homogeneous polynomials on the non convex $L_{\alpha}$ sphere given by

$$
K_{\alpha}:=\left\{(x, y) \in \mathbb{R}^{2}:|x|^{\alpha}+|y|^{\alpha} \leq 1\right\}, \quad 0<\alpha<1
$$

and verified that $f(x, y)$ is a uniform limit on $\partial K_{\alpha}$ of sums $h_{2 n}+h_{2 n+1}$ of homogeneous polynomials if and only if $f( \pm 1,0)=f(0, \pm 1)=0$. This means that the exceptional zero set of this domain is given by

$$
Z\left(K_{\alpha}\right)=\{( \pm 1,0),(0, \pm 1)\}, \quad 0<\alpha<1 .
$$

Clearly we can also rotate $K_{\alpha}$ by $\frac{\pi}{2}$ and consider the domain

$$
K_{\alpha}^{*}:=\left\{(x, y) \in \mathbb{R}^{2}:|x+y|^{\alpha}+|x-y|^{\alpha} \leq 2^{\alpha / 2}, \quad 0<\alpha<1,\right.
$$

for which the exceptional zero set consists of the four points

$$
Z\left(K_{\alpha}^{*}\right)=\{( \pm 1 / \sqrt{2}, \pm 1 / \sqrt{2})\}, \quad 0<\alpha<1 .
$$

Now consider the intersection of the above domains given by

$$
\Omega_{\alpha}:=K_{\alpha} \cap K_{\alpha}^{*}=\left\{(x, y) \in \mathbb{R}^{2}:|x|^{\alpha}+|y|^{\alpha} \leq 1 \text { and }|x+y|^{\alpha}+|x-y|^{\alpha} \leq 2^{\alpha / 2}\right\}, \quad 0<\alpha<1 .
$$

Evidently, $Z\left(K_{\alpha}\right) \cap K_{\alpha}^{*}=\emptyset$ and $Z\left(K_{\alpha}^{*}\right) \cap K_{\alpha}=\emptyset$. Thus it follows by Corollary 5 that $Z\left(\Omega_{\alpha}\right)=\emptyset$, and hence the homogeneous approximation property (??) holds for $\partial \Omega_{\alpha}$. It is interesting to note that the star like domain $\Omega_{\alpha}$ is "nowhere convex" in the sense that discs of arbitrarily small radius centered at any point of $\partial \Omega_{\alpha}$ have a non convex intersection with the interior of the domain. Thus we obtain "nowhere convex" star like domains which nevertheless satisfy the required approximation property.

B. Bodies of revolution. As it was mentioned in Section 2 circular cones are not covered by the known sufficient conditions for density of homogeneous polynomials. Applying Corollary 7 of the previous section for the simplex

$$
D=\left\{\mathbf{x} \in \mathbb{R}^{k}:\left|x_{1}\right|+\ldots+\left|x_{k}\right| \leq 1\right\}, k \geq 2
$$

which of course satisfies $Z(D)=\emptyset$ we obtain by Corollary 7 that the homogeneous approximation property (??) holds for circular cones which are obtained by a rotation of $D$ given by

$$
K_{D}=\left\{\mathbf{x} \in \mathbb{R}^{d}:\left|x_{1}\right|+\ldots+\left|x_{k-1}\right|+\left(x_{k}^{2}+\ldots+x_{d}^{2}\right)^{1 / 2} \leq 1\right\}, \quad d \geq k+1 .
$$

Furthermore, when $d=3$ it is easy to see that for any convex body of revolution in $\mathbb{R}^{3}$ the homogeneous approximation property (??) holds. This follows from the fact that (??) holds on the boundary of every convex 0-symmetric domain in $\mathbb{R}^{2}$ so applying Corollary 7 we obtain that the same is true for all convex bodies of revolution in $\mathbb{R}^{3}$, i.e., we have the next

Corollary 9 Let $D$ be any 0-symmetric convex body of revolution in $\mathbb{R}^{3}$. Then the homogeneous approximation property (??) holds on $\partial D$.

C. Exceptional zero sets of minimal cardinality. Since every exceptional zero set is symmetric with respect to the origin any nonempty exceptional zero set must consist of at least 2 points. Do there exist such minimal sets in $\mathbb{R}^{d}$ ? For the domain $K_{\alpha} \subset \mathbb{R}^{2}$ given by (??) its exceptional zero set $Z\left(K_{\alpha}\right)$ contains 4 points $( \pm 1,0),(0, \pm 1)$. Clearly rotating $K_{\alpha}$ and setting

$$
\Gamma_{\alpha}:=\left\{\mathbf{x} \in \mathbb{R}^{d}:\left|x_{1}\right|^{\alpha}+\left(x_{2}^{2}+\ldots+x_{d}^{2}\right)^{\alpha / 2} \leq 1\right\}, \quad 0<\alpha<1,
$$

it follows by Theorem 6 that

Now consider the ellipse

$$
Z\left(\Gamma_{\alpha}\right)=( \pm 1,0, \ldots, 0) \cup\left\{(0, \mathbf{y}), \mathbf{y} \in S^{d-2}\right\}
$$

$$
E:=\left\{\mathbf{x} \in \mathbb{R}^{d}: x_{1}^{2}+2\left(x_{2}^{2}+\ldots+x_{d}^{2}\right) \leq 1\right\}
$$

and its intersection with $\Gamma_{\alpha}$ given by

$$
\Theta_{\alpha}:=\left\{\mathbf{x} \in \mathbb{R}^{d}:\left|x_{1}\right|^{\alpha}+\left(x_{2}^{2}+\ldots+x_{d}^{2}\right)^{\alpha / 2} \leq 1 \text { and } x_{1}^{2}+2\left(x_{2}^{2}+\ldots+x_{d}^{2}\right) \leq 1\right\} .
$$

Since $Z(E)=\emptyset$ and $Z\left(\Gamma_{\alpha}\right) \cap E=( \pm 1,0, \ldots, 0)$ it follows by Theorem 4 that $Z\left(\Theta_{\alpha}\right)$ can contain only the pair of points $( \pm 1,0, \ldots, 0)$. Moreover similarly to [?], Proposition 5 it can be shown that $\{( \pm 1,0, \ldots, 0)\} \subset Z\left(\Theta_{\alpha}\right)$, we omit the details. Thus $Z\left(\Theta_{\alpha}\right)=\{( \pm 1,0, \ldots, 0)\}$ which provides the desired example of nonempty exceptional zero set consisting of 2 points. 


\section{References}

[1] D. Benko and A. Kroó, A Weierstrass-type theorem for homogeneous polynomials, Trans. Amer. Math. Soc., 361(2009), 1645-1665.

[2] R. A. DeVore and G. G. Lorentz, Constructive approximation, Grundlehren der mathematischen Wissenschaften, 303, Springer Verlag, 1993.

[3] K. G. Ivanov and V. Totik, Fast decreasing polynomials, Constr. Approx., 6 (1990), 1-20.

[4] A. Kroó and J. Szabados, On the density of homogeneous polynomials on regular convex surfaces, Acta Sci. Math., 75(2009), 143-159.

[5] A. Kroó and V. Totik, Density of bivariate homogeneous polynomials on non-convex curves (submitted)

[6] G. G. Lorentz, M. v. Golitschek and Y. Makovoz, Constructive Approximation - Advanced Problems, Springer (1996).

[7] A. Pinkus, Density in Approximation Theory, Surveys in Approximation Theory

[8] V. Totik, Approximation by homogeneous polynomials, J. Approx. Th., 174 (2013), 192-205.

[9] V. Totik, Weighted Approximation with Varying Weights, Lecture Notes in Mathematics, Springer (1991).

[10] P. Varjú, Approximation by homogeneous polynomials, Constr. Approx., 26,(2007), 317-337.

András Kroó

Alfréd Rényi Institute of Mathematics

Hungarian Academy of Sciences

Budapest, Hungary

and

Budapest University of Technology and Economics

Department of Analysis, Budapest, Hungary 